OPEN ACCESS

Edited by:

Qi Yuan,

Memorial University of Newfoundland, Canada

Reviewed by: Shan Huang, University of California, Los Angeles, United States

Amin Shakhawat,

Stanford University, United States

${ }^{*}$ Correspondence: Jin-Hui Wang

jhw@sun5.ibp.ac.cn

tThese authors have contributed equally to this work.

Received: 26 June 2017 Accepted: 26 September 2017 Published: 25 October 2017

Citation:

Lei Z, Wang D, Chen N, Ma K, Lu W, Song Z, Cui S and Wang J-H (2017) Synapse Innervation and Associative Memory Cell Are Recruited for Integrative Storage of Whisker and Odor Signals in the Barrel Cortex through miRNA-Mediated Processes. Front. Cell. Neurosci. 11:316. doi: 10.3389/fncel.2017.00316

\section{Synapse Innervation and Associative Memory Cell Are Recruited for Integrative Storage of Whisker and Odor Signals in the Barrel Cortex through miRNA-Mediated Processes}

\author{
Zhuofan Lei ${ }^{1,2,3 \dagger}$, Dangui Wang ${ }^{2 \dagger}$, Na Chen ${ }^{2 \dagger}$, Ke Ma ${ }^{1 \dagger}$, Wei $\mathrm{Lu}^{1,2+}$, Zhenhua Song ${ }^{1}$, \\ Shan Cui ${ }^{2}$ and Jin-Hui Wang ${ }^{1,2,3 *}$ \\ 'School of Pharmacy, Qingdao University, Dengzhou, China, ${ }^{2}$ Institute of Biophysics, Chinese Academy of Sciences, Beijing, \\ China, ${ }^{3}$ Department of Biology, University of Chinese Academy of Sciences, Beijing, China
}

Associative learning is a common way for information acquisition, and the integrative storage of multiple associated signals is essential for associative thinking and logical reasoning. In terms of the cellular mechanism for associative memory, our studies by behavioral task and cellular imaging demonstrate that paired whisker and odor stimulations lead to odorant-induced whisker motion and associative memory cell recruitment in the barrel cortex $(\mathrm{BC})$, which is driven presumably by synapse innervation from co-activated sensory cortices. To confirm these associative memory cells and synapse innervations essential for associative memory and to examine their potential mechanisms, we studied a causal relationship between epigenetic process and memory cell/synapse recruitment by manipulating miRNAs and observing the changes from the recruitments of associative memory cells and synapse innervations to associative memory. Anti-miRNA-324 and anti-miRNA-133a in the BC significantly downregulate new synapse innervation, associative memory cell recruitment and odorant-induced whisker motion, where Tau-tubulin kinase-1 expression is increased. Therefore, the upregulated miRNA-324 in associative learning knocks down Ttbk1-mediated Tau phosphorylation and microtubule depolymerization, which drives the balance between polymerization and depolymerization toward the axon prolongation and spine stabilization to initiate new synapse innervations and to recruit associative memory cells.

Keywords: learning, memory, synapse, memory cell, cortex, microRNA, Ttbk1

\section{INTRODUCTION}

Associative memory plays important roles in cognitive processes, such as logical reasoning and associative thinking (Kandel and Pittenger, 1999; Bailey et al., 2015; Wang and Cui, 2017). Animal models of conditioned reflexes (CR) have been used to elucidate mechanisms underlying associative learning and memory (Wasserman and Miller, 1997). Activity-dependent plasticity at synapses and neurons is associated with memory formation (Bliss and Collingridge, 1993; Blair et al., 2001; Maren, 2005; Karmarkar and Dan, 2006; Holtmaat and Svoboda, 2009; Silva et al., 2009). Associative memory cells are detected in the co-activated sensory cortices (Wang et al., 2015; Gao et al., 2016; Vincis and Fontanini, 2016) and their downstream brain areas (Naya et al., 2003; 
Takehara-Nishiuchi and McNaughton, 2008; Viskontas, 2008; Cai et al., 2016). Although new synapse innervation may constitute one of the driving forces to recruit associative memory cells, which are associated with associative memory (Wang et al., 2015, 2016, 2017; Gao et al., 2016; Wang and Cui, 2017), the causal relationship from new synapse innervation and associative memory cell recruitment to associative memory formation needs to be examined, as well as potential mechanisms underlying new synapse formation, associative memory cell recruitment and cross-modal associative memory remain to be addressed.

As the integration and storage of associated signals are induced by environmental information, epigenetics-mediated processes may be involved (Molfese, 2011; Kaas et al., 2013; Landry et al., 2013; Lattal and Wood, 2013; Woldemichael et al., 2014). The level of some miRNAs is altered during associative memory, especially the upregulation of miRNA-324 and miRNA-133a that influence actin filaments as well as microtubules for their polymerization (Yan et al., 2016). Since the balance between microtubule polymerization and depolymerization influences tubulin growth that plays a critical role in axon prolongation and spine stabilization (Dent and Kalil, 2001; Mitsuyama et al., 2009; Wilson et al., 2012; Ueda et al., 2015), we hypothesize that associative learning induces a chain reaction of miRNA expression change, microtubule polymerization, axon prolongation and synapse innervation for associative memory cell recruitment. Here, we aim to examine how miRNA-324/miRNA-133a and their regulated molecules serve to the recruitments of new synapse innervations and associative memory cells for the integration and storage of associated signals.

In terms of strategies to address new synapse innervation and associative memory cell formation for cross-modal associative memory by epigenetic process, the blockade of miRNA upregulation was performed by injecting miRNA antagomirs into the barrel cortex (BC). The molecular targets of miRNAs were studied by using dual luciferase report assay and westernblot. The detection of associative memory cells in the BC was done by using two-photon cell imaging in vivo. Synapse innervations were detected by using the neural tracing with fluorescent-tagged adeno-associated virus. In addition, the rationale for studying a role of epigenetic-mediated regulation in associative memory cell recruitment in the $\mathrm{BC}$ was based on our previous studies that this area was a primary location for crossmodal associative memory (Wang et al., 2015, 2016, 2017; Gao et al., 2016).

\section{MATERIALS AND METHODS}

All experiments were conducted based on the guideline by Administration Office of Laboratory Animals at Beijing China. Experiment protocols were approved by Institutional Animal Care Unit Committee in Administration Office of Laboratory Animals at Beijing China (B10831).

\section{Mouse Model of Associative Memory}

C57 Thy1-yellow fluorescent protein (YFP) mice (The Jackson Labs, USA, Feng et al., 2000) have been used in our study, in which certain amount of glutamatergic neurons was genetically labeled by YFP due to a weak promotion of Thyl in order to analyze cell-specific mechanism for associative memory. In our study, 54 male mice were used. In general, they were accommodated in the cages $(n=4$ mice per cage) with a usual circadian cycle about $12 \mathrm{~h}$ under light condition and $12 \mathrm{~h}$ under dark condition.

The two groups of mice in postnatal day 20 were treated by microinjecting the antagomirs of miRNA-324-5p/miRNA$133 \mathrm{a}-3 \mathrm{p}$ and the control of these antagomirs into barrel cortices, respectively. Forty-eight hours after the injection, these mice were trained by pairing mechanic whisker stimulus (WS) with odor stimulus (OS, butyl acetate toward their noses; Wang et al., 2015; Gao et al., 2016; Yan et al., 2016; Guo et al., 2017; Liu et al., 2017; Zhao et al., 2017). The concentration of butyl acetate was $99.99 \%$ in analytic purity. The paired WS and OS were given by a multiple-sensory modal stimulator (ZL201410499466), in which the intensities, time and intervals of OS and WS were precisely set. The OS intensity was sufficient to induce the activity of olfactory bulb neurons, and the WS intensity was sufficient to induce whisker fluctuation after WS ended. Each of the mice was trained $20 \mathrm{~s}$ each time, five times per day with $2 \mathrm{~h}$ of intervals for 10 days. During the training, each mouse was placed in a home-made cage. In entire experimental procedures, these mice were not experiencing stressful condition and circadian disturbance. In addition, the mice have normal whisking and symmetric whiskers (Wang et al., 2015; Gao et al., 2016; Yan et al., 2016; Guo et al., 2017).

Whisker motion tracks were monitored by a digital video camera (240 fps) and were quantified in onset latency from odor test to whisker motion, whisking frequency and whisking duration (the software self-programed in Matlab; and ImageJ, version. 1.47, the National Institute of Health, Rockville, MD, USA). Whisking frequency and duration were converted into digital signals in $0.5 \mathrm{~s}$ as one unit, such that their dynamic values per second were presented before, during and after odor-test. The responses of mouse whiskers to the odor-test (butyl acetate, $20 \mathrm{~s}$ ) were measured at the end of each training day to quantify the onset time and levels of the CR. CR-formation was defined to meet the following criteria. The patterns of odorant-induced whisker motion were similar to those of WS-induced whisker motion. Whisking frequency and whisker retraction time were significantly increased as well as the latency of whisker motion in response to odor-test was significantly reduced, compared with control and before the training. As this type of whisker motion induced by odorant was originally induced by WS, the odor signal evoked a recall of whisker signal and led to whisker motion (Wang et al., 2015; Gao et al., 2016; Yan et al., 2016; Guo et al., 2017).

Long whiskers (such as arcs 1-2) on the same side and rows were assigned for mechanical stimulations and for the observations during the odor-test. This selection was based on the studies of cross-modal plasticity (Ni et al., 2010; Ye et al., 2012; Zhang et al., 2013). We did not trim the shorter whiskers since whisker trimming elevated the excitability of the BC (Zhang et al., 2013). 


\section{The Injection of miRNA Antagomirs into the Barrel Cortex}

miRNA antagomirs are the chemically modified cholesterolconjugated single-stranded RNA analogs complementary to specific miRNAs. For instance, miRNA-324-5p antagomir is a RNA analog complementary to miRNA-324-5p. miRNA-133a$3 p$ antagomir is analog to miRNA-133a-3p. Antagomir control is miRNA fragment from C. elegans, since it is not functional in mammalians and has been used as the control of miRNA antagomirs (Sun et al., 2013; Wang et al., 2013). Antagomir for miRNA-324-5p (5'Cy3), antagomir for miRNA-133a-3p $\left(5^{\prime} \mathrm{Cy} 3\right)$ and antagomir control were synthesized by RiboBio Company (Guangzhou, China). The mice were anesthetized by the intraperitoneal injection of urethane $(1.5 \mathrm{~g} / \mathrm{kg})$ and fixed in a stereotaxic apparatus. The microinjections were fulfilled by imbedding the gage needles $(0.3 \mathrm{~mm}$ in diameters $)$ on the surface of the barrel cortices and by giving the pressure with the microsyringe (RWD Life Science, Shenzhen, China). One nanomolar antagomirs including anti-miRNA324 and antimiRNA-133a in a ratio of one to one or antagomir control were dissolved in $2 \mu \mathrm{l}$ of the ACSF, and were injected into barrel cortices in the mice, in which three time of injections were given to each mouse with 3 days of intervals (i.e., injections on day 1, day 4 and day 7) and each injection was slowly done about $30 \mathrm{~min}$. It is noteworthy that as the retention volume of the injection microneedle is about $1 \mu \mathrm{l}$ and the delivery velocity of the microinjection is $0.03 \mu \mathrm{l} / \mathrm{min}$, the local diffusion of the injected solution makes the actual volume into the injection site of the $\mathrm{BC}$ being less than $2 \mu \mathrm{l}$, such that the side-effect of this microinjection is minimized. The transfected area of the microinjection without injury is showed in Figure 1B. In addition, the transfection rate of miRNA antagomirs and their control in barrel cortices was $50 \%$ on average in our study (Supplementary Figure S6), which has been considered in analyses of axon projection and synapse innervation.

\section{Fluorescence Labeling}

The mice were anesthetized by intraperitoneal injections of urethane $(1.5 \mathrm{~g} / \mathrm{kg})$. In surgical operations, the anesthetic depth was set at the levels where the mice demonstrated lack of reflexes in pinch withdrawal and eyelid blinking. The body temperature was maintained by a heating blanket whose temperature was electronically controlled at $37^{\circ} \mathrm{C}$. $\mathrm{Ca}^{2+}$-sensitive dye, Oregon Green BAPTA-1-AM (OGB-1, Invitrogen, USA), was used to measure neuronal activities. A craniotomy $(2 \mathrm{~mm})$ was made on the skull above barrel cortical areas $(1 \mathrm{~mm}$ posterior
A

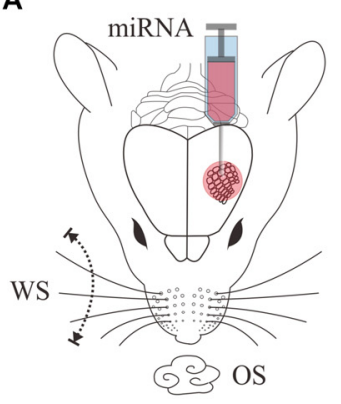

B

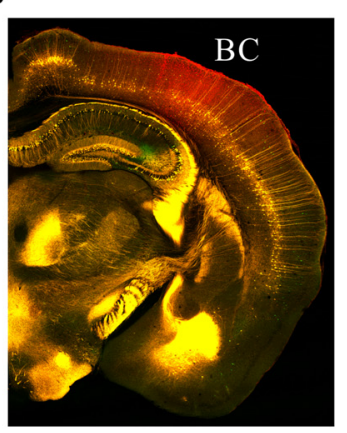

c
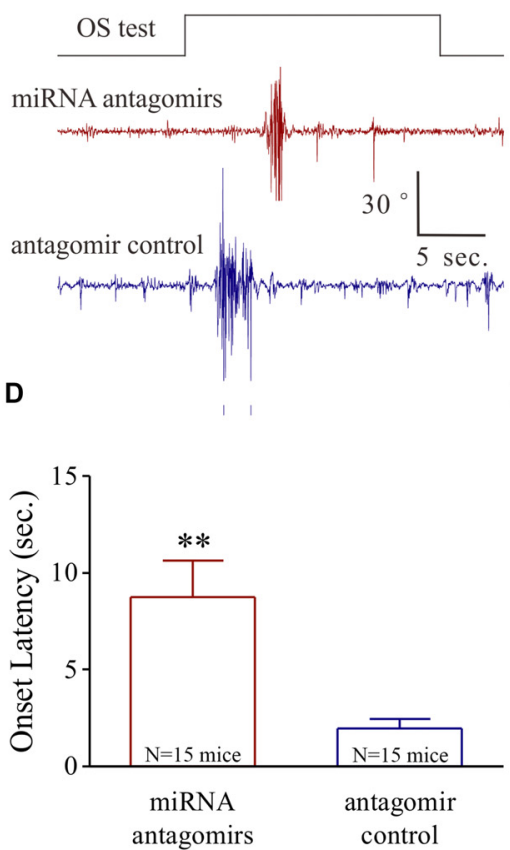

E
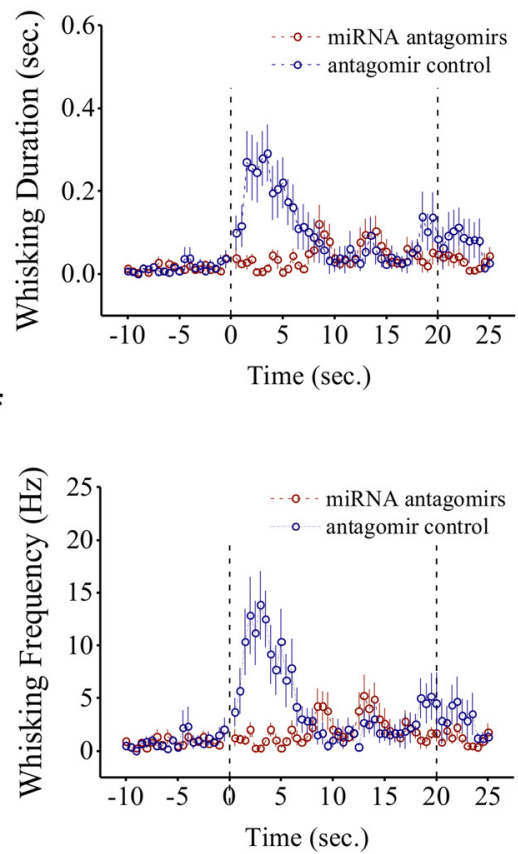

FIGURE 1 | Odorant-induced whisker motion is impaired by anti-miRNA-324-5p and anti-miRNA-133a-3p. (A) These miRNA antagomirs and their controls were injected into the barrel cortices of two groups of the mice, respectively. The mice then were trained by pairing whisker stimulus (WS) and odor stimulus (OS) for 10 days. (B) After their behavioral tests, the correct injections of miRNA antagomirs (conjugated with red fluorescent) were examined in the barrel cortices. (C) shows the impaired whisker motions in response to OS-test from a mouse in antagomir group (red trace) and a mouse in control group (blue). Calibration bars are 30 degrees of whisker retraction and $5 \mathrm{~s}$. (D) Latencies from OS onset to whisker motions are $8.75 \pm 1.89 \mathrm{~s}$ in antagomir group ( $n=15$; red bar) and $1.96 \pm 0.47 \mathrm{~s}$ in controls ( $p=0.005, n=15$; blue, one-way ANOVA). (E) shows whisking durations from the mice in antagomir group (red symbols) and in control (blue). (F) shows whisking frequencies in the mice of antagomir group (red symbols) and in control (blues). The frequency and duration of whisker motion are calculated by whisker motion per $0.5 \mathrm{~s}$ in all of the time phases. ${ }^{* *} p<0.01$. 
to the Bregma and $3.5 \mathrm{~mm}$ lateral to the midline; Paxinos and Watson, 2005). The dura was intact in experiments. The detailed information about surgical operation, dye loading and post-treatment for cell imaging can be referred in our studies (Zhao et al., 2012; Wang et al., 2015).

\section{Two-Photon Cell Imaging}

The anesthetic depth for doing in vivo two-photon cell imaging in the mice was set at the moderate reflexes in pinch withdrawal and eyelid blinking, as well as the responses of their whiskers to the test stimuli (Zhao et al., 2012; Wang et al., 2015). $\mathrm{Ca}^{2+}$ imaging was done $1 \mathrm{~h}$ after dye injections under a two-photon microscope (Olympus FV-1000, Tokyo, Japan), which was equipped by the two-photon laser-beam generator (Mai Tai, Physical Spectrum, USA) and mounted to an upright microscope (Olympus BX61WI) with the water immersion objectives (IR-LUMPLan Fl, $40 \times, 0.8 \mathrm{NA}$ ). A wave of two-photon laser beam at $810 \mathrm{~nm}$ was given to excite $\mathrm{OGB}$ and examine neuronal responses to WS and OS. The emission wavelength was $525 \mathrm{~nm}$ for $\mathrm{Ca}^{2+}$. binding OGB. Average power delivered to the barrel cortices was less than $75 \mathrm{~mW}$ to minimize photo bleach. Whole field images were acquired at $10 \mathrm{~Hz}$ of frame rate $(256 \times 256$ pixels). The parameters for photomultiplier tube (PMT) and laser beam were locked in the measurements throughout all experiments to have consistency in data comparison.

Similar to the stimuli in behavioral task, an odor-test pulse toward the noses or mechanical pulses to the whiskers on the contralateral side of two-photon imaged cortices were used to induce cell responses. Stimulus patterns were pair-pulses (OS vs. WS or WS vs. OS) and pulse intervals were 60 s (Wang et al., 2015; Yan et al., 2016).

\section{Imaging Data Analyses}

Cellular $\mathrm{Ca}^{2+}$ fluorescence signals in response to stimuli were acquired by Fluoview-10 software (Olympus Inc., Japan) and analyzed in cell bodies by NIH ImageJ and MATLAB (MathWorks). To reduce photon and PMT noise, a median filter (radius, 1 pixel) was used to all images. $\mathrm{Ca}^{2+}$ signals were normalized and presented as relative fluorescence changes $(\Delta \mathrm{F} / \mathrm{F})$. Basal fluorescence $(\mathrm{F})$ was an averaged value before stimuli. Values about $\Delta \mathrm{F}$ were the differences of $\mathrm{Ca}^{2+}$ signal fluorescent strengths between evoked response and basal condition (Zhao et al., 2012). All fluorescence signals were subtracted from the noise signals of unstained blood vessels. Normalized $\mathrm{Ca}^{2+}$ signals were smoothed by low-pass Butterworth filter to remove low-level fluctuation and to minimize distortions from $\mathrm{Ca}^{2+}$ transient (Yaksi and Friedrich, 2006). The effective signals from each of active cells were judged based on a criterion that their relative fluorescence changes were greater than 2.5 times of standard deviation of baseline values lasting for $500 \mathrm{~ms}$ (Wang et al., 2015). The magnitudes of $\mathrm{Ca}^{2+}$ transient signals, i.e., activity strength, were measured from the point at 2.5 times of standard deviation of baseline values to their peaks. The durations of $\mathrm{Ca}^{2+}$ transient signals were measured as the time from the point at 2.5 times of standard deviation of baseline values in the rising phase to the point at 2.5 times of standard deviation of baseline values in the decay phase. The data were presented as mean \pm SEM (Zhang et al., 2012; Zhao et al., 2012; Wang et al., 2015).

With these recorded $\mathrm{Ca}^{2+}$ signals, we analyzed their strengths and durations of the barrel cortical neurons in response to WS and OS (Wang et al., 2015). The strength of $\mathrm{Ca}^{2+}$ signal was proportional to the spike frequency, and the duration of $\mathrm{Ca}^{2+}$ signals was proportional to number of spikes (Petersen et al., 2005; Yaksi and Friedrich, 2006). It is noteworthy that fluorescent signals are measured one time, but not repetitively, in order to prevent their photo bleach and effects on signal strength analyses.

\section{Neural Tracing and Synapse Formation}

The structural connections between cortical regions were traced by injecting pAAV-SynaptoTag-Cherry-GFP (a gift from Dr. Tom Südhof) into the piriform cortex (PC) and by detecting its presence in the BC from C57 Thy1-YFP mice (Zhang et al., 2013) whose glutamatergic neurons were genetically labeled by YFP. The working principle of this AAV was that Synapsin-I promoter initiates the expression of EGFP-synaptobrevin-2 in presynaptic boutons and terminals as well as the expression of mCherry in the neuron (Xu and Südhof, 2013), especially in cell body (Figure 3A). In pAAV injection, the glass pipettes were positioned in the PC $(1.0 \mathrm{~mm}$ posterior to the Bregma, $3.5 \mathrm{~mm}$ lateral to midline and $4.75 \mathrm{~mm}$ in depth), based on the map of the mouse brains (Paxinos and Watson, 2005). Two weeks after injecting pAAV into their cortices, the axon projection and synapse formation were analyzed.

The mice were anesthetized by intraperitoneal injections of sodium pentobarbital, and perfused by $4 \%$ paraformaldehyde in $0.1 \mathrm{M}$ phosphate buffer solution (PBS) into left ventricle until their bodies were rigid. The brains were quickly isolated and fixed in $4 \%$ paraformaldehyde PBS for additional $24 \mathrm{~h}$. The cerebral brains were sliced in a series of coronal sections at $100 \mu \mathrm{m}$. These slices were rinsed by PBS for three times, air-dried and coverslipped. In order to clearly show three-dimension images for new synapses in the $\mathrm{BC}$, we placed the brain slices into a solution (Sca/eA2) for a few hours to make them transparent (Hama et al., 2011).

The images to show synaptic contacts between GFP-labeled axon boutons and YFP-labeled glutamatergic neuron spines in the barrel cortices were used to count the new synapses. The images for YFP-labeled glutamatergic neurons and GFP-labeled axonal boutons in cortical layers II-III were photographed under this confocal microscopy with oil lens (Plan Apo VC 60× 1.4NA; Nikon A1R plus). The excite wavelength was $488 \mathrm{~nm}$ for GFP and YFP. Although the peaks of the GFP and YFP emission wavelengths are closely at $510 \mathrm{~nm}$ and $525 \mathrm{~nm}$, respectively, we scanned the images of these neurons by setting up the optical grating in 505-515 $\mathrm{nm}$ for GFP and the optical grating in $545-555 \mathrm{~nm}$ for YFP to separate their fluorescent images. These images were merged to construct the newly formed synapses. Although we have reported the functional connection from the PC to the BC in CR-formation mice (Wang et al., 2015), it is noteworthy that these synaptic contacts are examined by seeing their functions in spines/boutons and their labeling with synapsemarker proteins. In this regard, we would call these synapse 
contacts as "presumed new synapses", though they are stated as "newly formed synapses" in the text of our article. In the confocal imaging, the resolution was $0.05 \mu \mathrm{m}$ per pixel, the minimal pixels for the measured spines were at least 9-10 in the line. The structure and the density of the newly formed synapses were analyzed in layers II-III of the barrel cortices by applying softwares Image (version. 1.47; The National Institute of Health, MD, USA) and Imaris (version 7.2.3; Bitplane, England).

In the analyses of the dendritic spines, axon terminals and the synapses, we defined the contacts as the synapses based on the separations between presynaptic and postsynaptic identities less than $0.1 \mu \mathrm{m}$. We calculated synaptic contacts per millimeter dendrites. Moreover, as the YFP did not label all of the glutamatergic neurons due to the weak Thyl promoter, synapsinGFP-labeled presynaptic boutons might innervate the spines on non-YFP glutamatergic neurons, such that the densities of GFP-labeled boutons per $\mathrm{mm}^{3}$ were also calculated to assess the number of new synapses.

\section{Dual Luciferase Reporter Assay}

The entire procedure of Dual Luciferase Reporter Assay was given in our previous publication (Ma et al., 2016). The $3^{\prime}$-untranslated region (UTR) sequence of Ttbk1 was amplified and fused into the XhoI and NotI sites of the dual luciferase vector psiCHECK2, a generous gift from Dr. Xue (Institute of Biophysics, Chinese Academy of Sciences). The site-mutation of the detected miRNA targeting site of $3^{\prime}$-UTR fragment was constructed based on a guideline of QuikChange Lighting Site-Directed Mutagenesis Kit (Stratagene, La Jolla, CA, USA). miRNA mimics and their negative control (NC) were synthesized by Guangzhou Rui Bo Biological Technology Co. Ltd., China. Primer sequences are listed in Supplemenatry Table S1. For the luciferase reporter detection, HEK293T cells were planted in RPMI media containing $10 \%$ fetal bovine serum at $5 \times 10^{4}$ cells per well in 24-well plates. After $24 \mathrm{~h}$, these cells were co-transfected with $20 \mathrm{ng}$ psiCHECK2-3'UTR wild-type or mutant reporter plasmids. In the meantime, these wells of cell culture were added by $50 \mathrm{nM}$ of miRNA mimic or miRNA-NC by using Lipofectamine 2000 transfection reagent (Invitrogen, Carlsbad, CA, USA). The activities of firefly and Renilla luciferases were assessed after 48 h by using Dual-Glo ${ }^{\circledR}$ Luciferase Assay System (Promega, Cat. E2920, USA), based on the manufacturer's protocols. A ratio of Renilla luciferase activity to firefly luciferase activity was used to analyze the level of mRNA expressions regulated by miRNAs, such as mRNA downregulation or upregulation (Parsons et al., 2000). Each treatment was performed in the triplicates from three independent experiments.

\section{Western Blot}

Barrel cortical tissues from mice whose barrel cortices received the injections of miRNA-324-5p/miRNA-133a-5p antagomirs or antagomir control were gently washed three times in ice-cold PBS and then placed in $1 \mathrm{ml}$ of RIPA Lysate buffer with PMSF (Beyotime Biotechnology, China) for their homogenizations. The homogenized tissues were removed into a new EP tube $(1.5 \mathrm{ml})$, kept at $4^{\circ} \mathrm{C}$ in a refrigerator for $30 \mathrm{~min}$, and centrifuged at $12,000 \mathrm{~g} / \mathrm{min}$ for $15 \mathrm{~min}$. The concentration of total proteins in supernatant liquid was measured by using the protein assay based on manufacturer instruction. Fifty micrograms of total proteins per sample was then resolved in $10 \%$ sodium dodecyl sulfate polyacrylamide gel electrophoresis (SDSPAGE). After SDS-PAGE, proteins were electrically transferred onto nitrocellulose membrane. The membrane was incubated by blocking solution ( $1 \times$ TBS; $0.1 \%$ Tween $20 ; 5 \%$ non-fat milk) at room temperature $\left(25^{\circ} \mathrm{C}\right)$ for $2 \mathrm{~h}$, and then incubated overnight by a primary antibody (1:2000 in dilution) of Ttbk1 (ab103944, Abcam) or by primary antibody (1:1000 in dilution) of $\beta$-actin (AC004, ABclonal Technology) in 5\% milk in TBST. After incubations with their corresponding secondary antibodies conjugated with peroxidase (Beyotime Biotechnology, China), the bands of these proteins were visualized by using the enhanced chemiluminescence ECL Plus immunoblotting detection system (Climx Science Instruments Co. Ltd., China). The protein bands corresponding to the expected size were selected and read by using this computerized scanner. The pixel density in each of these bands was determined by this computer after the background was corrected, which were used to quantify for the relative level of these proteins. The optical densities of each band relative to measured values of $\beta$-actin bands were determined using ImageJ software (Zhu et al., 2017).

\section{Statistical Analyses}

The paired $t$-test was used in the comparisons of the experimental data before and after associative learning as well as the neuronal responses to WS and odorant stimulus in each of the mice. One-way ANOVA was used for the statistical comparisons in the changes of neuronal activity and morphology between the miRNA antagomir and antagomir control groups. It is noteworthy that the behavioral studies and the data analyses are conducted by different individuals.

\section{RESULTS}

In the present study, we aim to study the causal relationship from new synapse innervations and associative memory cell recruitment to associative memory as well as to examine their molecular mechanisms by manipulating epigenetic process. To investigate the roles of epigenetics-mediated events, such as miRNAs, in associative memory cell recruitment, synapse innervation and associative memory, we have injected the antagomirs of miRNA-324-5p and miRNA-133a-3p or antagomir NC into mouse barrel cortices (Figures 1A,B). The selection of these two anti-miRNAs was based on our observation that miRNA-324 and miRNA-133a, which presumably regulate axon growth and synapse formation, were upregulated in barrel cortices from mice that expressed odorant-induced whisker motion (Yan et al., 2016). In some mice from these two groups, the injection of pAAV-SynaptoTag-mCherry-GFPs (Xu and Südhof, 2013) into the piriform cortices and the detection of its presence in the barrel cortices were conducted to examine the effect of anti-miRNA on synapse formation. These mice were subsequently trained by pairing the OS and the WS for 10 days (Wang et al., 2015; Gao et al., 2016). 
After the training in the mice, the OS-test was given to their noses. The mice that have received antagomir control express whisker motion in response to the OS (Figure 1C). This odorant-induced whisker motion appears to be impaired in the mice that have received the antagomirs of miRNA-324 and miRNA-133a (Figure 1C). Latencies from the OS onset to whisker motion is prolonged in antagomir group, compared with antagmir control (Figure 1D). Whisking durations (Figure 1E) and frequencies (Figure 1F) in response to the OS are lowered in antagomir group than antagomir control. In addition, the changes in whisking amplitude and bouts duration are given in Supplementary Figure S1. Thus, anti-miRNA-324 and antimiRNA-133a downregulate odorant-induced whisker motion, i.e., miRNA-324 and miRNA-133a are required for associative memory.

The influence of anti-miRNAs on the recruitment and activities of associative memory cells was examined in the barrel cortices of WS/OS-trained mice by using two-photon cell $\mathrm{Ca}^{2+}$ imaging, which had been injected with the antagomirs of miRNA-324 and miRNA 133a (Figure 2A) or antagomir control (Figure 2B). Figures 2C,D show the digitized traces of cellular $\mathrm{Ca}^{2+}$ signals in response to the WS vs. the OS from a mouse in antagomir group and from a mouse in antagomir control group. The proportions of the neurons in response to the OS are significantly lower in antagomir group than antagomir control (Figure 2E), implying that anti-miRNA-324/-133a attenuate associative memory cell recruitment. To OS-responsive cells, $\mathrm{Ca}^{2+}$ signal strengths (Figure 2F) and durations (Figure 2G) are significantly lower in antagomir group than antagomir control. However, $\mathrm{Ca}^{2+}$ signals in response to the WS are not statistically different between antagomir group and antagomir control group (Figures 2E-G). Our results indicate that anti-miRNA-324 and anti-miRNA-133a downregulate the conversion of barrel cortical neurons into associative memory cells, as well as their activity strength and duration.

This indication should be further tested in barrel cortical neurons that are transfected vs. non-transfected by miRNA antagomirs in mice whose barrel cortices receive the injection of miRNA antagomirs. In barrel cortices, red fluorescentlabeled neurons were thought to be antagomir-transfected neurons, or vice versa, since miRNA antagomirs had been conjugated with $\mathrm{Cy} 3$. The proportions of miRNA antagomirtransfected neurons are $48.2 \pm 5.2 \%$ in response to the WS and $6.9 \pm 1.0 \%$ in response to the OS (Figure $2 \mathbf{H}$ ), indicating the less effect of anti-miRNA on WS-responses. To OS-responsive cells, $73.3 \pm 8.8 \%$ of them appear to have no antagomirtransfection and $26.7 \pm 8.8 \%$ of them antagomir-transfection (Figure 2I). $\mathrm{Ca}^{2+}$ signal strength and duration in response to the OS are significantly lower in antagomir-transfected cells than non-transfected cells (Figure 2J,K). These parameters are not changed in the cells transfected by antagomir control (Supplementary Figure S2). This result supports the indication that anti-miRNA-324 and anti-miRNA-133a downregulate the recruitment of associative memory cells and their activities by reducing their responses to the OS but not the WS. In other words, miRNA-324 and miRNA-133a are required for recruiting associative memory cells.
The recruitment of associative memory cells in the BC may be driven by the synapse innervations from the PC (Wang et al., 2015; Gao et al., 2016), which we have tested by the injection of pAAV-SynaptoTag-Cherry-GFP into the PC (red arrow in Figure 3A) and the detection of its presence in the BC (green, Supplementary Figure S3). After the WS/OS-paired training in these mice, axon projections from the $\mathrm{PC}$ to the $\mathrm{BC}$ are detected in antagomir control, but less in antagomir group (Figure $\mathbf{3 A}$ ). The strength of the projected axons is statistically lower in antagomir group than antagomir control group (Figure 3B). Synapsin I-labeled boutons in the BC are statiscially lower in antagomir group than antagomir control group (Figures 3C,D). The synapses that are the contacts between GFP-labeled presynaptic boutons and YFP-labeled postsynaptic spines on glutamatergic neurons in the BC appear low in antagomir group, compared to those in antagomir control group (Figure 3E), which is statistically different (Figure 3F). In addition, the changes in spine density and volume are showed in Supplementary Figure S4. Thus, anti-miRNA-324 and antimiRNA-133a downregulate synapse innervations from the PC to the $\mathrm{BC}$ during associative memory. That is, miRNA-324 and miRNA-133a are required for new axon projection and synapse formation.

Taken these results together, we suggest that there are the causal relationships among synapse formation, associative memory cell recruitment and associative memory, in which the epigenetic molecular events triggered by miRNA-324 and miRNA-133a play critical roles in these processes.

mRNA Ttbk1 that translates Tau-tubulin kinase- 1 is a predicted target of these miRNAs. This brain-specific kinase phosphorylates microtubule-associated protein Tau, leading to its aggregation (Sato et al., 2006; Xu et al., 2010). Taken this with our studies that miRNA-324 is upregulated (Yan et al., 2016) and Ttbk1 is downregulated (Supplementary Figure S5) in barrel cortices of CR-formation mice, we have examined whether miRNA-324-5p directly acts on Ttbk1 by dual luciferase report assay. Luciferase reporter plasmids including the wild-type or mutant 3'-UTRs of predicted miRNA-324-5p binding site in Ttbk1 (top panel in Figure 4A) were transfected into HEK293T cells. After miRNA-324-5p or its NC is added into cell cultural dishes for $24 \mathrm{~h}$, the relative activity of luciferase reporter for Ttbk1 $3^{\prime}$-UTR is significantly inhibited by miRNA-324 and this inhibition is reversed by mutating miRNA-324-5p binding site (Figure 4A). That is, Ttbk1 is a target of miRNA-324-5p.

The influence of anti-miRNAs on the expression of Ttbk1 protein was examined by western-blot. The proteins were harvested from the barrel cortices that had received the injection of miRNA antagomirs or their controls. The levels of Ttbk1 appear higher in antagomir group than in antagomir control, which is statistically different (Figure 4B). Memory cell recruitment and synapse formation in associative memory are influenced by downregulating Tau-tubulin kinase- 1 .

\section{DISCUSSION}

Pairing whisker and odor signals in the mice leads to odorant-induced whisker motion alongside natural whisker- 
A

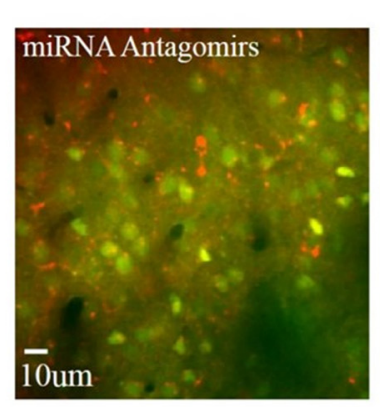

E

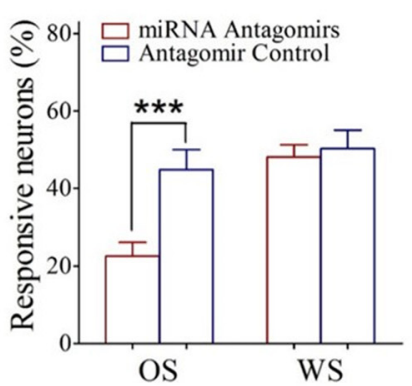

B

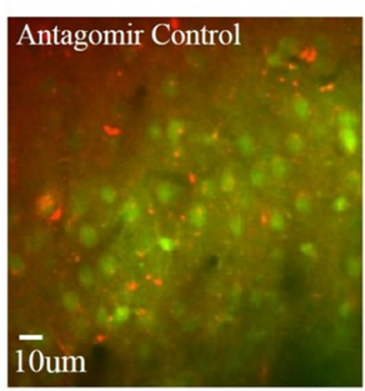

F

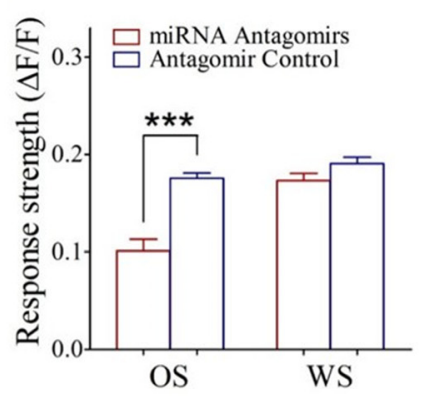

c
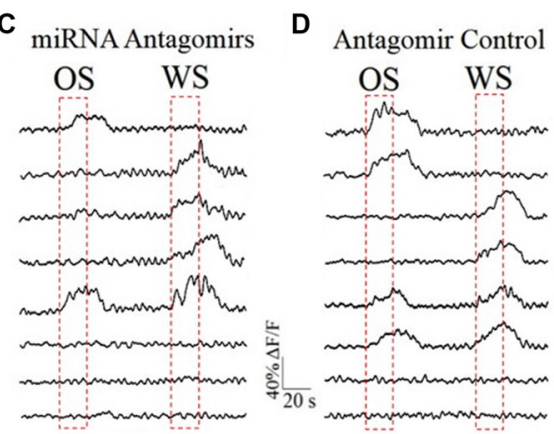

G

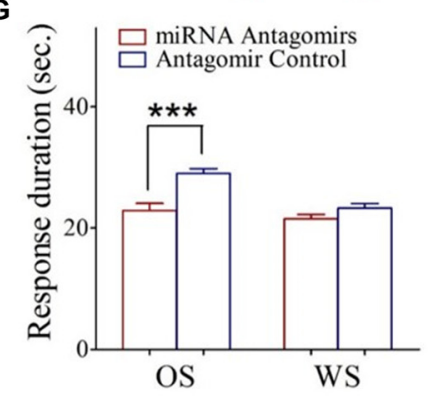

H

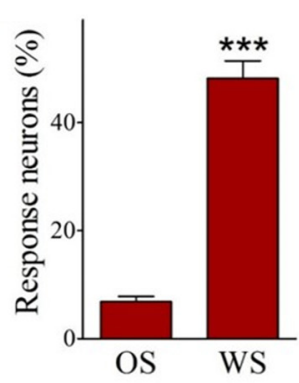

I

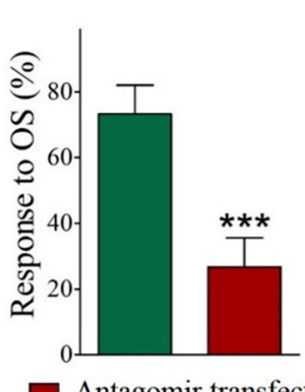

J

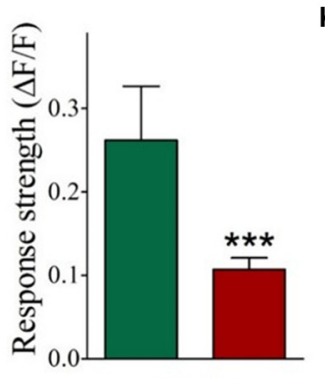

K

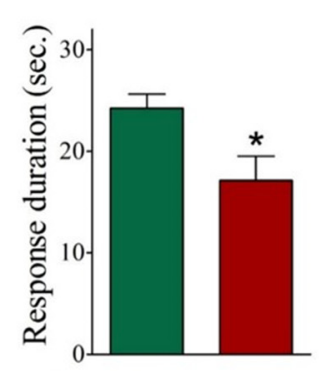

miRNA Antagomirs $\mathrm{n}=6$ mice

Antagomir non-transfected neurons

Antagomir Control $\mathrm{n}=6$ mice

FIGURE 2 | The responses of barrel cortical neurons to OS are downregulated by the antagomirs of miRNA-324-5p/miRNA-133a-3p. (A) shows the Ca ${ }^{2+}$ images in the neurons from a mouse in antagomir group. As miRNA antagomir is conjugated by red fluorescent Cy3, yellow cells are thought to be miRNA antagomir transfected neurons. (B) shows the $\mathrm{Ca}^{2+}$ images in the neurons from a mouse in control. (C) illustrates the digitized $\mathrm{Ca}^{2+}$ signals from the neurons in response to OS and WS from a mouse of antagomir group. (D) shows the digitized $\mathrm{Ca}^{2+}$ signals from the neurons in response to OS and WS from a mouse of controls. (E) The percentages of the neurons in response to OS are $22.60 \pm 3.50 \%$ of fluorescence-detected neurons from antagomir group $(n=6$ mice, red bar) and $44.80 \pm 5.20 \%$ in control group $\left(p=0.003, n=6\right.$ mice; blue). (F) To OS-responsive neurons, $\mathrm{Ca}^{2+}$ signal strengths are $0.1 \pm 0.01$ in antagomir group ( $n=49$ cells; red bar) and $0.18 \pm 0.005$ in controls ${ }^{* * *} p<0.001, n=141$ cells; blue bar). (G) $\mathrm{Ca}^{2+}$ signal durations are $22.90 \pm 1.20 \mathrm{~s}$ in antagomir group ( $n=49$ cells; red bar) and $29.0 \pm 0.80$ s in controls ${ }^{* * *} p<0.001, n=141$ cells; blue bar). $(\mathbf{H})$ shows the percentages of antagomir-transfected neurons in response to the OS (8 out of 116 cells) and to the WS (56 out of 116) in antagomir group. (I) shows the percentages of the neurons in response to the OS that are antagomir-transfected (red-filled bar) and non-antagomir-transfected (green-filled) from antagomir group mice. (J) $\mathrm{Ca}^{2+}$ signal strengths in response to $\mathrm{OS}$ are $0.26 \pm 0.06$ in non-transfected neurons ( $n=41$ cells, green bar) and $0.11 \pm 0.01$ in antagomir-transfected neurons $\left({ }^{* * *} p<0.001, n=8\right.$ cells; red) from antagomir group mice. (K) $\mathrm{Ca}^{2+}$ signal durations in response to OS are $24.25 \pm 1.39 \mathrm{~s}$ in non-transfected neurons ( $n=41$ cells; green bar) and $17.10 \pm 2.40 \mathrm{~s}$ in antagomir-transfected neurons $\left(p=0.03, n=8\right.$ cells; red) from antagomir group mice. ${ }^{*} p<0.05$.

induced whisker motion, i.e., cross-modal associative memory. After receiving new synapse innervations from the axons of co-activated piriform cortical neurons, the barrel cortical neurons are recruited to be associative memory cells that encode the newly learnt odor signal alongside the innate whisker signal. These processes are attenuated by anti-miRNA-324 and antimiRNA-133a (Figures 1-3). By studying a causal relationship between anti-miRNA and attenuated cellular events, our study indicates that epigenetic-regulated processes induce new synapse innervation, associative memory cell recruitment and associative memory. The associated activations of the barrel and piriform cortices by pairing whisker and odor signals produce their mutual synapse innervations and associative memory cells for the storage of associated signals. Our data reveals a novel mechanism, i.e., new synapse innervation and associative memory cell recruitment primarily in the sensory cortices 
A

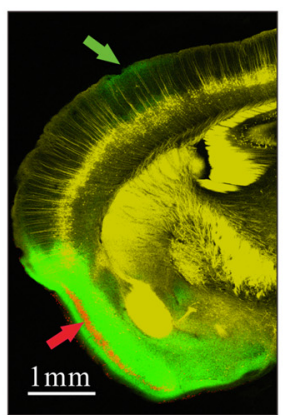

antagomir

control

C

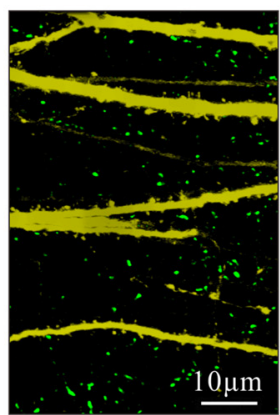

antagomir

control

E

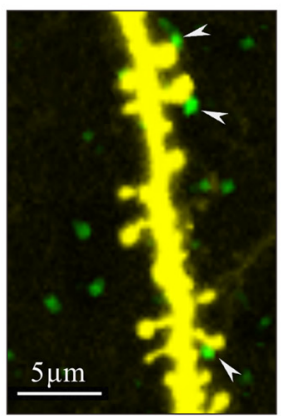

antagomir

control

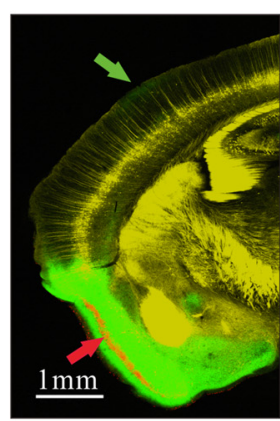

miRNA

antagomirs

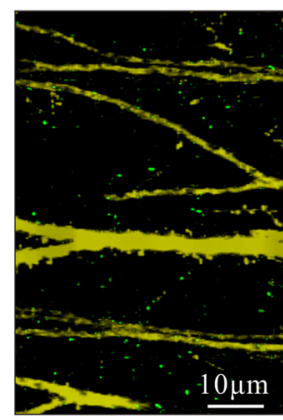

miRNA

antagomirs

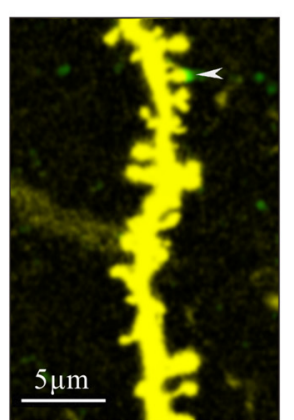

miRNA

antagomirs

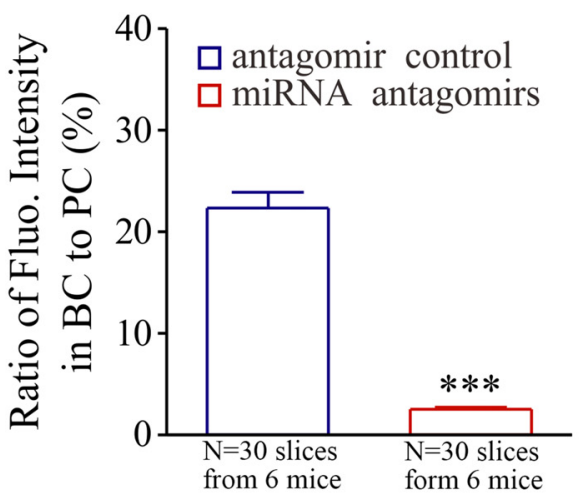

D
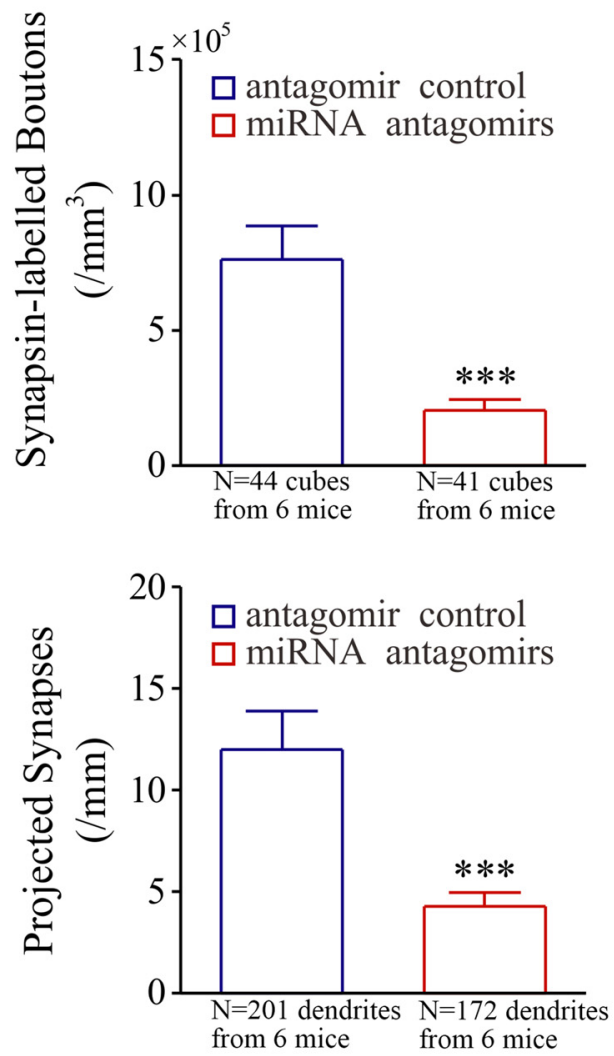

FIGURE 3 | Synaptic innervations from the piriform cortex $(\mathrm{PC})$ to the barrel cortex (BC) after their co-activation are downregulated by the antagomirs of miRNA-324-5p/miRNA-133a-3p. Neural tracing is done by injecting pAAV-SynaptoTag-Cherry-GFP into the PC and by detecting its presence in the BC.

(A) illustrates neural tracings in the mice from control group (left panel) and antagomir group (right). Red arrow shows injection site and green arrow is the projected site. (B) Based on the ratio of green fluorescent intensity in BC to red fluorescent intensity of neuronal somata in PC, the strengths of the projected axons are $2.50 \pm 0.20 \%$ in antagomir group (red bar, $n=30$ slices from 6 mice) and $22.40 \pm 1.50 \%$ in control (blue bar; **** $p<0.001, n=30$ slices from 6 mice). (C) shows synapsin I-labeled boutons in the barrel cortices from control mice (left panel) and antagomir groups (right panel). (D) The densities of synapsin I-labeled boutons are $2.0 \pm 0.4 \times 10^{5}$ per $\mathrm{mm}^{3}$ in antagomir group (red bar; $n=41$ cubes) and $7.60 \pm 1.27 \times 10^{5} / \mathrm{mm}^{3}$ in controls (blue; **** $p<0.001, n=44$ ). (E) shows synapse contacts (white arrows) between presynaptic boutons (green) and postsyanptic spines (yellow) on dendrites (per mm) in the barrel cortices from mice in control (left panel) and antagomir groups (right). (F) The number of new synapses on apical dendrties are $4.70 \pm 0.70$ per $\mathrm{mm}$ in antagomir group (red bar; $n=172$ dendrites) and $11.90 \pm 1.90$ per $\mathrm{mm}$ in control group (blue; ${ }^{* * *} p<0.001, n=201$ ).

for associative memory, beyond previous focuses in synaptic plasticity.

In our study, miRNA-324 acts and downregulates mRNA Ttbk1 that guides the expression of Tau-tubulin kinase-1, which is supported by the data of dual luciferase report assay and western-blot in Figure 4. This kinase is able to phosphorylate microtubule-associated protein Tau (Sato et al., 2006; Xu et al., 2010). Phosphorylated 
A
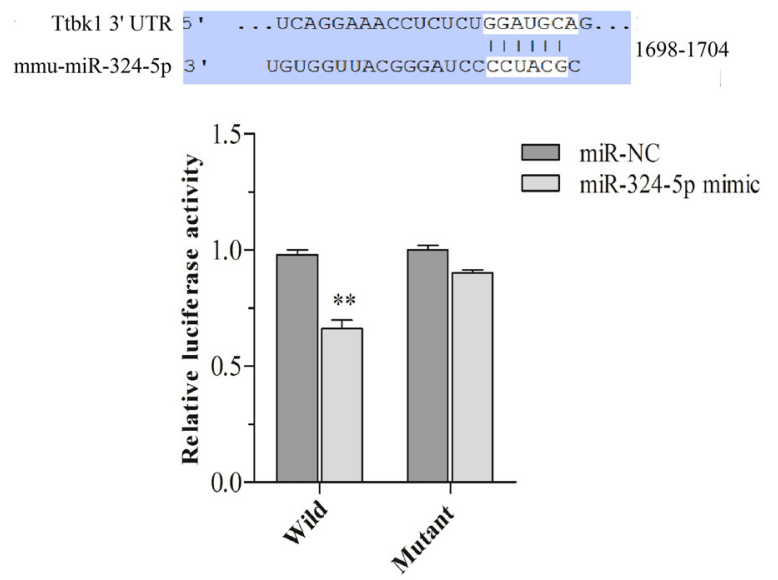

B Ttbk1 (143 Kda)

$\beta$-Actin (43 Kda)

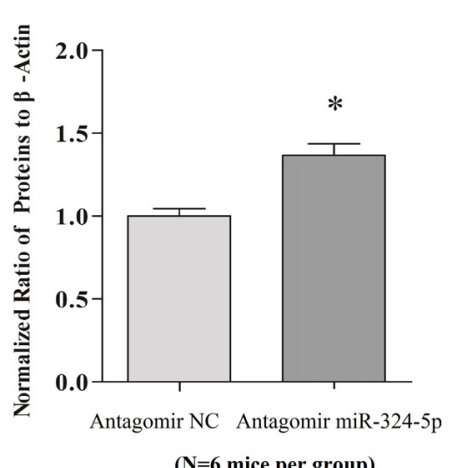

FIGURE 4 | miRNA-324-5p acts onto Ttbk1 and downregulates Tau-tubulin kinase-1. (A) Luciferase reporter assay is done by the co-transfection of luciferase reporter containing wild or mutant 3'-untranslated region (UTR) of Ttbk1 with miRNA-324-5p mimic or its negative control (NC) into HEK293T cells. Luciferase activity was read $48 \mathrm{~h}$ after co-transfection. Top panel shows the predicted interaction between wild-type $3^{\prime}-$ UTR of Ttbk1 and miRNA-324-5p. The relative activities of luciferase reporter for Ttbk1 $3^{\prime}$-UTR are $1.0 \pm 0.05$ in the group of adding NC (dark-gray bar) and $0.66 \pm 0.07$ in the group of adding miRNA-324-5p mimic $\left(p=0.006, n=24\right.$; light-gray bar). The relative activities of luciferase reporter for $3^{\prime}$-UTR of Ttbk1 with mutant miRNA-324-5p binding site are $1.0 \pm 0.06$ in the group of NC (dark-gray bar) and $0.92 \pm 0.05$ in the group of miRNA-324-5p (light-gray). The ratio for NC is normalized as 1. (B) The expression of Tau-tubulin kinase-1 was studied by western-blot. The ratios of Ttbk1 to $\beta$-actin are $1.0 \pm 0.1$ in antagomir control mice (light-gray bar, $n=6$ ) and 1.37 \pm 0.07 in miRNA-324-5p antagomir mice (dark-gray, $p=0.02, n=6$ ). The ratios for control mice are normalized as $1 .{ }^{*} p<0.05 ;{ }^{* *} p<0.01$.

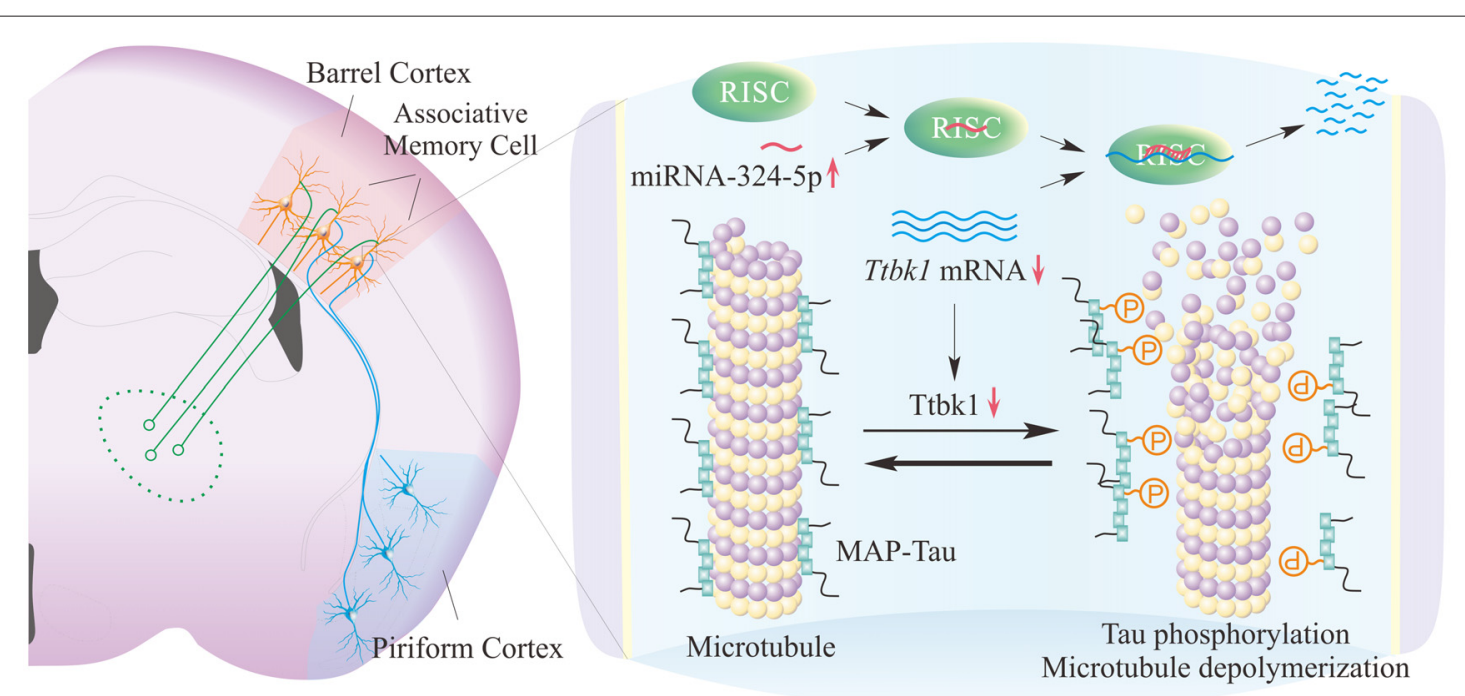

FIGURE 5 | miRNA-324-reguated Ttbk1 presumably plays a crucial role in the recruitment of synapse innervation and associative memory cells in barrel cortices. Left panel shows the synapse innervation on barrel cortical neurons from piriform cortical neurons and the recruitment of associative memory cells in the BC. Right panel shows the potential molecular mechanisms underlying the recruitments of synapse innervation and associative memory cells. The upregulated miRNA-324 downregulates mRNA Ttbk1 and protein Ttbk1, which reduces the phosphorylation of microtubule-associated protein Tau (MAP-Tau) by shifting the balance between MAP-Tau phosphorylation and dephosphorylation toward dephosphorylated MAP-Tau. These dephosphorylated Tau can stabilize microtubules and facilitate axon growth from the PC as well as synapse and associative memory cell recruitments in the BC.

Tau proteins induce their aggregations and destabilize microtubules, which lead to microtubule depolymerization, axon degeneration and spine deformation (Sánchez et al., 2000). The downregulation of Ttbk1 by the upregulated miRNA-324 may shift the balance between axon prolongation and retardation toward axon growth from the $\mathrm{PC}$ to the $\mathrm{BC}$, and trigger axon terminals to make new synapses onto barrel cortical neurons. These new synapses may drive these barrel cortical neurons to be recruited as associative memory cells (Figure 5). 
The cellular targets of miRNA-mediated epigenetic process for associative memory include the following. Both presynaptic axon boutons (Figure 3) and postsynaptic spines (Supplementary Figure S4) are upregulated in their densities and size, which influence the chance to form new synapse innervations. The recruitment of associative memory cells is upregulated, since anti-miRNA-324/133a reduces the number of associative memory cells as well as their response strength and duration to the new odor signal (Figures 2E-K). As miRNA-324/133a are predicted to regulate the axon prolongation and synapses based on bioinformatics (Yan et al., 2016), our results in vivo about axon prolongation and synapse formation are consistent with molecular profiles.

There are two forms of neural plasticity that are associated with memory formation and cognition, i.e., the recruitments of new synapse innervations and associative memory cells as well as the functional change of synapses and neurons. Plasticity in our study falls into new synapse formation and associative memory cell recruitment that are driven by the association of new and innate signals via miRNA-mediated processes. These associative memory cells are specific for the integration and storage of the newly acquired signal and innate signal. On the other hand, plasticity at those existing synapses in a specific pathway, such as LTP (Bliss and Lomo, 1973) and LTD (Stanton and Sejnowski, 1989), may not be related to the integration and storage of associated signals. These activity-dependent potentiation and depression at the synapses after their formation may work for the process whether their innervated neurons are able to be activated for signal retrieval and memory presentation. In this regard, our study about associative memory cells bring insight into cellular mechanisms for memory formation.

Based on our studies, the characteristics of associative memory cell are proposed below. They are recruited to encode multiple associated signals. They receive multiple synapse innervations from the sensory cortices that are co-activated and encode different signals. Their axons project toward the brain areas related to cognitions, emotions and behaviors

\section{REFERENCES}

Bailey, C. H., Kandel, E. R., and Harris, K. M. (2015). Structural components of synaptic plasticity and memory consolidation. Cold Spring Harb. Perspect. Biol. 7:a021758. doi: 10.1101/cshperspect.a021758

Blair, H. T., Schafe, G. E., Bauer, E. P., Rodrigues, S. M., and LeDoux, J. E. (2001). Synaptic plasticity in the lateral amygdala: a cellular hypothesis of fear conditioning. Learn. Mem. 8, 229-242. doi: 10.1101/lm.30901

Bliss, T. V., and Collingridge, G. L. (1993). A synaptic model of memory: long-term potentiation in the hippocampus. Nature 361, 31-39. doi: 10.1038/ $361031 \mathrm{a} 0$

Bliss, T., and Lomo, T. (1973). Long-lasting potentiation of synaptic transmission in the dentate area of the anaesthetized rabbit following stimulation of the perforant path. J. Physiol. 232, 331-356. doi: 10.1113/jphysiol.1973. sp010273

Cai, D. J., Aharoni, D., Shuman, T., Shobe, J., Biane, J., Song, W., et al. (2016). A shared neural ensemble links distinct contextual memories encoded close in time. Nature 534, 115-118. doi: 10.1038/nature 17955

Dent, E. W., and Kalil, K. (2001). Axon branching requires interactions between dynamic microtubules and actin filaments. J. Neurosci. 21, 9757-9769. for memory retrieval. The recruitment of associative memory cells is initiated by epigenetic-mediated process. The number of recruited associative memory cells is proportional to memory strength and maintenance. The downregulations of the associative memory cells and their new synapse innervations reduce memory capacity (Wang et al., 2015; Gao et al., 2016; Wang and Cui, 2017). The working principle of these associative memory cells in cross-modal associative memory may be based on the facilitation of their activity strengths by newly innervated synapses from the co-activated sensory cortices. For instance, barrel cortical neurons receive synapse innervations from the PC. These synapse activities induced by OS can drive these barrel cortical neurons toward a threshold of firing spikes, and their spikes further activate downstream cortical neurons for whisker motion and other relevant behaviors.

\section{AUTHOR CONTRIBUTIONS}

ZL, DW, NC, KM, WL and ZS contributed to experiments and data analyses. SC contributed to draw diagram. J-HW worked for the concept, project design and article writing.

\section{ACKNOWLEDGMENTS}

We thank Dr. Tom Südhof for pAAV-SynaptoTag-mCherryGFP, and Yuanchao Xue for dual luciferase vector psiCHECK2. We also thank Kim Davis for proof reading before revision. This study is supported by National Basic Research Program (2013CB531304 and 2016YFC1307101) and Natural Science Foundation China (81671071 and 81471123) to J-HW.

\section{SUPPLEMENTARY MATERIAL}

The Supplementary Material for this article can be found online at: https://www.frontiersin.org/articles/10.3389/fncel. 2017.00316/full\#supplementary-material

Feng, G., Mellor, R. H., Bernstein, M., Keller-Peck, C., Nguyen, Q. T., Wallace, M., et al. (2000). Imaging neuronal subsets in transgenic mice expressing multiple spectral variants of GFP. Neuron 28, 41-51. doi: 10.1016/s0896-6273(00) 00084-2

Gao, Z., Chen, L., Fan, R., Lu, W., Wang, D., Cui, S., et al. (2016). Associations of unilateral whisker and olfactory signals induce synapse formation and memory cell recruitment in bilateral barrel cortices: cellular mechanism for unilateral training toward bilateral memory. Front. Cell. Neurosci. 10:285. doi: 10.3389/fncel.2016.00285

Guo, R., Ge, R., Zhao, S., Liu, Y., Zhao, X., Huang, L., et al. (2017). Associative memory extinction is accompanied by decayed plasticity at motor cortical neurons and persistent plasticity at sensory cortical neurons. Front. Cell. Neurosci. 11:168. doi: 10.3389/fncel.2017.00168

Hama, H., Kurokawa, H., Kawano, H., Ando, R., Shimogori, T., Noda, H., et al. (2011). Scale: a chemical approach for fluorescence imaging and reconstruction of transparent mouse brain. Nat. Neurosci. 14, 1481-1488. doi: 10.1038/ nn. 2928

Holtmaat, A., and Svoboda, K. (2009). Experience-dependent structural synaptic plasticity in the mammalian brain. Nat. Rev. Neurosci. 10, 647-658. doi: $10.1038 / \mathrm{nrn} 2699$ 
Kaas, G. A., Zhong, C., Eason, D. E., Ross, D. L., Vachhani, R. V., Ming, G. L., et al. (2013). TET1 controls CNS 5-methylcytosine hydroxylation, active DNA demethylation, gene transcription and memory formation. Neuron 79, 1086-1093. doi: 10.1016/j.neuron.2013.08.032

Kandel, E. R., and Pittenger, C. (1999). The past, the future and the biology of memory storage. Philos. Trans. R. Soc. Lond. B Biol. Sci. 354, 2027-2052. doi: 10.1098/rstb.1999.0542

Karmarkar, U. R., and Dan, Y. (2006). Experience-dependent plasticity in adult visual cortex. Neuron 52, 577-585. doi: 10.1016/j.neuron.2006. 11.001

Landry, C. D., Kandel, E. R., and Rajasethupathy, P. (2013). New mechanisms in memory storage: piRNAs and epigenetics. Trends Neurosci. 36, 535-542. doi: 10.1016/j.tins.2013.05.004

Lattal, K. M., and Wood, M. A. (2013). Epigenetics and persistent memory: implications for reconsolidation and silent extinction beyond the zero. Nat. Neurosci. 16, 124-129. doi: 10.1038/nn.3302

Liu, Y., Gao, Z., Chen, C., Wen, B., Huang, L., Ge, R., et al. (2017). Piriform cortical glutamatergic and GABAergic neurons express coordinated plasticity for whisker-induced odor recall. Oncotarget doi: 10.18632/oncotarget.21207 [Epub ahead of Print].

Ma, K., Xu, A., Cui, S., Sun, M., Xue, Y., and Wang, J.-H. (2016). Impaired GABA synthesis, uptake and release are associated with depression-like behaviors induced by chronic mild stress. Transl. Psychiatry 6:e910. doi: 10.1038/ tp.2016.181

Maren, S. (2005). Synaptic mechanisms of associative memory in the amygdala. Neuron 47, 783-786. doi: 10.1016/j.neuron.2005. 08.009

Mitsuyama, F., Futatsugi, Y., Okuya, M., Karagiozov, K., Peev, N., Kato, Y., et al. (2009). Amyloid beta: a putative intra-spinal microtubule-depolymerizer to induce synapse-loss or dentritic spine shortening in Alzheimer's disease. Ital. J. Anat. Embryol. 114, 109-120.

Molfese, D. L. (2011). Advancing neuroscience through epigenetics: molecular mechanisms of learning and memory. Dev. Neuropsychol. 36, 810-827. doi: 10.1080/87565641.2011.606395

Naya, Y., Yoshida, M., and Miyashita, Y. (2003). Forward processing of long-term associative memory in monkey inferotemporal cortex. J. Neurosci. 23, 2861-2871.

Ni, H., Huang, L., Chen, N., Zhang, F., Liu, D., Ge, M., et al. (2010). Upregulation of barrel GABAergic neurons is associated with cross-modal plasticity in olfactory deficit. PLoS One 5:e13736. doi: 10.1371/journal.pone. 0013736

Parsons, S. J., Rhodes, S. A., Connor, H. E., Rees, S., Brown, J., and Giles, H. (2000). Use of a dual firefly and Renilla luciferase reporter gene assay to simultaneously determine drug selectivity at human corticotrophin releasing hormone 1 and 2 receptors. Anal. Biochem. 281, 187-192. doi: 10.1006/abio. 2000.4570

Paxinos, G., and Watson, C. (2005). The Mouse Brain: in Stereotaxic Coordinates. Amsterdam, Boston: Elsevier Academic Press.

Petersen, O. H., Michalak, M., and Verkhratsky, A. (2005). Calcium signalling: past, present and future. Cell Calcium 38, 161-169. doi: 10.1016/j.ceca. 2005.06.023

Sánchez, C., Diaz-Nido, J., and Avila, J. (2000). Phosphorylation of microtubuleassociated protein 2 (MAP2) and its relevance for the regulation of the neuronal cytoskeleton function. Prog. Neurobiol. 61, 133-168. doi: 10.1016/s03010082(99)00046-5

Sato, S., Cerny, R. L., Buescher, J. L., and Ikezu, T. (2006). Tau-tubulin kinase 1 (TTBK1), a neuron-specific tau kinase candidate, is involved in tau phosphorylation and aggregation. J. Neurochem. 98, 1573-1584. doi: 10.1111/ j.1471-4159.2006.04059.x

Silva, A. J., Zhou, Y., Rogerson, T., Shobe, J., and Balaji, J. (2009). Molecular and cellular approaches to memory allocation in neural circuits. Science 326, 391-395. doi: 10.1126/science.1174519

Stanton, P. K., and Sejnowski, T. J. (1989). Associative long-term depression in the hippocampus induced by hebbian covariance. Nature 339, 215-218. doi: $10.1038 / 339215 \mathrm{a} 0$

Sun, Y., Li, Q., Gui, H., Xu, D. P., Yang, Y. L., Su, D. F., et al. (2013). MicroRNA124 mediates the cholinergic anti-inflammatory action through inhibiting the production of pro-inflammatory cytokines. Cell Res. 23, 1270-1283. doi: $10.1038 / \mathrm{cr} .2013 .116$
Takehara-Nishiuchi, K., and McNaughton, B. L. (2008). Spontaneous changes of neocortical code for associative memory during consolidation. Science 322, 960-963. doi: 10.1126/science.1161299

Ueda, H., Zhou, J., Xie, J., and Davis, M. M. (2015). Distinct roles of cytoskeletal components in immunological synapse formation and directed secretion. J. Immunol. 195, 4117-4125. doi: 10.4049/jimmunol. 1402175

Vincis, R., and Fontanini, A. (2016). Associative learning changes cross-modal representations in the gustatory cortex. Elife 5:e16420. doi: 10.7554/eLife. 16420

Viskontas, I. V. (2008). Advances in memory research: single-neuron recordings from the human medial temporal lobe aid our understanding of declarative memory. Curr. Opin. Neurol. 21, 662-668. doi: 10.1097/WCO. 0b013e3283168e 03

Wang, J.-H., and Cui, S. (2017). Associative memory cells: formation, function and perspective. F1000Research 6:283. doi: 10.12688/f1000research. 11096.1

Wang, J.-H., Feng, J., and Lu, W. (2017). Associative memory cells are recruited to encode triple sensory signals via synapse formation. Biophys. J. 112, 443a-444a. doi: 10.1016/j.bpj.2016.11.2377

Wang, X., Guo, B., Li, Q., Peng, J., Yang, Z., Wang, A., et al. (2013). miR214 targets ATF4 to inhibit bone formation. Nat. Med. 19, 93-100. doi: 10.1038/ $\mathrm{nm} .3026$

Wang, J.-H., Wang, D., Gao, Z., Chen, N., Lei, Z., Cui, S., et al. (2016). Both glutamatergic and gabaergic neurons are recruited to be associative memory cells. Biophys. J. 110. Suppl. 481a. doi: 10.1016/j.bpj.2015. 11.2571

Wang, D., Zhao, J., Gao, Z., Chen, N., Wen, B., Lu, W., et al. (2015). Neurons in the barrel cortex turn into processing whisker and odor signals: a cellular mechanism for the storage and retrieval of associative signals. Front. Cell. Neurosci. 9:320. doi: 10.3389/fncel.2015.00320

Wasserman, E. A., and Miller, R. R. (1997). What's elementary about associative learning? Annu. Rev. Psychol. 48, 573-607. doi: 10.1146/annurev.psych. 48.1.573

Wilson, S. M., Xiong, W., Wang, Y., Ping, X., Head, J. D., Brittain, J. M., et al. (2012). Prevention of posttraumatic axon sprouting by blocking collapsin response mediator protein 2 -mediated neurite outgrowth and tubulin polymerization. Neuroscience 210, 451-466. doi: 10.1016/j.neuroscience.2012. 02.038

Woldemichael, B. T., Bohacek, J., Gapp, K., and Mansuy, I. M. (2014). Epigenetics of memory and plasticity. Prog. Mol. Biol. Transl. Sci. 122, 305-340. doi: 10.1016/b978-0-12-420170-5.00011-8

Xu, J., Sato, S., Okuyama, S., Swan, R. J., Jacobsen, M. T., Strunk, E., et al. (2010). Tau-tubulin kinase 1 enhances prefibrillar tau aggregation and motor neuron degeneration in P301L FTDP-17 tau-mutant mice. FASEB J. 24, 2904-2915. doi: 10.1096/fj.09-150144

Xu, W., and Südhof, T. C. (2013). A neural circuit for memory specificity and generalization. Science 339, 1290-1295. doi: 10.1126/science. 1229534

Yaksi, E., and Friedrich, R. W. (2006). Reconstruction of firing rate changes across neuronal populations by temporally deconvolved $\mathrm{Ca}^{2+}$ imaging. Nat. Methods 3, 377-383. doi: 10.1038/nmeth874

Yan, F., Gao, Z., Chen, P., Huang, L., Wang, D., Chen, N., et al. (2016). Coordinated plasticity between barrel cortical glutamatergic and GABAergic neurons during associative memory. Neural Plast. 2016:5648390. doi: $10.1155 / 2016 / 5648390$

Ye, B., Huang, L., Gao, Z., Chen, P., Ni, H., Guan, S., et al. (2012). The functional upregulation of piriform cortex is associated with cross-modal plasticity in loss of whisker tactile inputs. PLoS One 7:e41986. doi: 10.1371/journal.pone. 0041986

Zhang, G., Gao, Z., Guan, S., Zhu, Y., and Wang, J. H. (2013). Upregulation of excitatory neurons and downregulation of inhibitory neurons in barrel cortex are associated with loss of whisker inputs. Mol. Brain 6:2. doi: 10.1186/17566606-6-2

Zhang, F., Liu, B., Lei, Z., and Wang, J. (2012). mGluR1,5 activation improves network asynchrony and GABAergic synapse attenuation in the amygdala: implication for anxiety-like behavior in DBA/2 mice. Mol. Brain 5:20. doi: $10.1186 / 1756-6606-5-20$ 
Zhao, X., Huang, L., Guo, R., Liu, Y., Zhao, S., Guan, S., et al. (2017). Coordinated plasticity among glutamatergic and GABAergic neurons and synapses in the barrel cortex is correlated to learning efficiency. Front. Cell. Neurosci. 11:221. doi: 10.3389/fncel.2017.00221

Zhao, J., Wang, D., and Wang, J.-H. (2012). Barrel cortical neurons and astrocytes coordinately respond to an increased whisker stimulus frequency. Mol. Brain 5:12. doi: 10.1186/1756-6606-5-12

Zhu, Z., Wang, G., Ma, K., Cui, S., and Wang, J. H. (2017). GABAergic neurons in nucleus accumbens are correlated to resilience and vulnerability to chronic stress for major depression. Oncotarget 8, 35933-35945. doi: 10.18632/oncotarget.16411
Conflict of Interest Statement: The authors declare that the research was conducted in the absence of any commercial or financial relationships that could be construed as a potential conflict of interest.

Copyright (c) 2017 Lei, Wang, Chen, Ma, Lu, Song, Cui and Wang. This is an open-access article distributed under the terms of the Creative Commons Attribution License (CC BY). The use, distribution or reproduction in other forums is permitted, provided the original author(s) or licensor are credited and that the original publication in this journal is cited, in accordance with accepted academic practice. No use, distribution or reproduction is permitted which does not comply with these terms. 